\title{
Therapeutic implication of BAL in patients with neutropenia
}

\author{
Dagmar Kuehnhardt • Manja Hannemann • \\ Bernd Schmidt • Ulrike Heider • Kurt Possinger • \\ Jan Eucker
}

Received: 15 August 2008 / Accepted: 14 April 2009/Published online: 5 May 2009

(C) Springer-Verlag 2009

\begin{abstract}
Bronchoalveolar lavage (BAL) is a practicable procedure establishing the etiology of pneumonia. In patients with neutropenia, empirical antimicrobial treatment is mandatory immediately after diagnosis of infection, usually before results of BAL are available. We evaluated the impact of BAL on treatment and outcome of pneumonia in immunocompromised patients with a special regard to neutropenia. Bronchoscopy with BAL was performed in 58 episodes of clinical documented pneumonia in patients with hematological malignancies $(88 \%)$ or solid tumors $(12 \%)$, in 30 cases patients had neutropenia, in 28 cases patients had no neutropenia. In $93 \%$ of cases, BAL was performed under empirical antimicrobial treatment. BAL fluid was cultivated for bacteria, fungi, and tested for Pneumocystis jirovecii and cytomegalovirus (CMV). BAL revealed positive bacterial results in $67 \%$ of cases. Gram-positive microorganisms were detected in $95 \%$ of positive BAL results, gram-negative microorganisms in $23 \%$, mixed bacterial cultures occurred in $41 \%$. Positive fungi cultures were found in 59\%. P. jirovecii was detected in 5\% of cases tested and CMV in $8 \%$. There was no significant difference between neutropenic and non-neutropenic patients. BAL results directed a change of therapy in only six of 58 episodes (5\%). Overall mortality related to pneumonia was $16 \%$. In this patient setting, the yield of BAL rarely has a
\end{abstract}

D. Kuehnhardt $\cdot$ M. Hannemann $\cdot$ U. Heider $\cdot$ K. Possinger $\cdot$

J. Eucker $(\bowtie)$

Department of Oncology and Hematology,

University Hospital Charité,

10117 Berlin, Germany

e-mail: jan.eucker@charite.de

B. Schmidt

Department of Pulmonology, University Hospital Charité, Berlin, Germany significant influence on treatment and outcome of pneumonia. The early beginning of antimicrobial treatment reduces the diagnostic yield of BAL. In patients with pneumonia during neutropenia, its use should be well considered.

Keywords BAL $\cdot$ Pneumonia $\cdot$ Neutropenia

\section{Introduction}

Immunocompromised patients constitute a heterogenous group and include patients with hematological malignancies and solid tumors, patients undergoing chemotherapy, patients after autologous and allogeneic bone marrow transplantation or patients after solid organ transplantation, as well as patients with primary immunodeficiency or acquired immunodeficiency. In patients with malignant disease, one of the most important known risk factors for infections is neutropenia. Several studies have proven the risk and analyzed the duration of neutropenia as a special risk factor $[1,6,10]$. Infections during neutropenia are a major cause of complications and mortality in patients treated with chemotherapy [23]. Among these infections, pneumonia is a very serious complication and has a poor prognosis, especially if treatment is delayed [10, 11, 31]. Patients with neutropenia should be treated immediately after onset of fever or any sign of infection and antimicrobial treatment should be initiated according to published guidelines even if positive diagnostic results are still lacking [13, 16, 18, 19].

Bronchoalveolar lavage (BAL) is a helpful procedure to establish diagnosis and clarify the etiology of pneumonia. It has been shown in patients with different kinds of immunosuppression that BAL may provide a diagnostic yield in 13 to $60 \%$ of cases $[2,8,14,15,24,25,29]$; but 
there exists only a few data concerning BAL in patients with pneumonia during neutropenia $[3,5,9,11,21,27,30]$. Maschmeyer et al. [19] recommended BAL in febrile neutropenic patients not responding to empirical antimicrobial therapy, in case of positive chest radiography findings. Bronchoscopy with BAL is considered as a safe procedure [21], but especially in severely ill patients, this intervention is associated with a high complication rate [2]. The long-term benefit of this procedure is difficult to demonstrate.

It is a question if BAL should be used frequently in immunocompromised patients with pneumonia or if its use should be restricted to special questions like the detection of Pneumocystis jirovecii pneumonia, cytomegalovirus (CMV) pneumonia, or tuberculosis.

To determine the diagnostic yield and therapeutic implication of BAL in patients with hematological diseases and solid tumors, we reviewed the experience of our Cancer Center with a special focus to neutropenia.

\section{Materials and methods}

\section{Characteristics}

We studied retrospectively the patients who underwent bronchoscopy and BAL during six consecutive years (1999-2004) in the Department of Hematology and Oncology of the university hospital Charité, Berlin. The study comprised 58 episodes of documented pneumonia in patients with hematological malignancies and solid tumors who underwent BAL. The study evaluated retrospectively the influence of diagnostic bronchoscopy with BAL on treatment and outcome of pneumonia in patients with and without neutropenia. Biopsies were not included in the analysis. We reviewed 86 charts; in 27 cases, bronchoscopy was performed due to non-infectious reasons and was excluded. One episode of pneumonia was not evaluated and excluded because of a relevant lack of clinical, microbiological, or radiographic reports.

From these 58 evaluable cases, 30 episodes occurred during neutropenia (group A) and 28 episodes occurred in non-neutropenic patients (group B). A patient was evaluated as neutropenic if at time of diagnosis of pneumonia, neutropenia $<1,000 / \mu 1$ was present. Patient characteristics are shown in Table 1.

The diagnosis of pneumonia was based on clinical symptoms and laboratory signs of infection (fever $>38^{\circ} \mathrm{C}$, purulent sputum, C-reactive protein), new or progressive infiltrates on the chest radiograph and on high-resolution computed tomography (CT). Empirical antimicrobial treatment was begun promptly after onset of infection signs according to published guidelines $[13,16]$. Prior to BAL, in case of any sign of infection including fever of unknown origin, samples were taken from suspected infectious site before antimicrobial treatment was started. A thorough physical examination was performed and a chest radiograph was taken. If chest radiograph revealed pulmonary changes which were not highly suspicious on pneumonia, highresolution CT was performed to help distinguish pneumonia from other pulmonary diseases. Pneumonia was unlikely if CT scan revealed a negative result as high-resolution CT has a high negative predictive value [12].

\section{BAL and microbiological methods}

BAL was performed under mild sedation (midazolam) and local anesthesia (lidocaine) after overnight fasting. We used fiber-optic bronchoscopes with a 2.0-2.2-mm working channel (Olympus, Pentax) via the transoral approach. Bronchoalveolar lavage was performed by wedging the tip of the bronchoscope into the segmental or sub-segmental bronchus in the area with the greatest radiologic abnormality. When bilateral changes were present, BAL was performed in the middle lobe. Sterile saline $(150 \mathrm{~mL}$; $0.9 \%$ ) warmed to body temperature was instilled in $20-\mathrm{mL}$ aliquots. Gentle manual suction with a syringe was applied to retrieve the saline. In case of respiratory failure, the amount of fluid was reduced to $80 \mathrm{~mL}$. Bronchoalveolar lavage fluid was collected and immediately carried to the laboratory. Contraindications for bronchoscopy and BAL were bleeding abnormalities, acute asthma, or respiratory insufficiency (hypercapnia $>44 \mathrm{mmHg}$, hypoxia $<60 \mathrm{mmHg}$ ), severe cardiac disease or if clinical assessment indicated that oxygen and hemodynamic status might be compromised by the procedure.

Cases of pneumonia were evaluable if BAL fluid was at least processed for microscopic examination, bacterial and fungal culture. Samples were analyzed microscopically after staining with Gram stain and calcofluor white fluorescence stain for the detection of microorganisms. We did not focus on the evaluation of the cytologic pattern of BAL since it was shown in other investigations that the therapeutic usefulness of cytological examination was very low in hematological patients with chemotherapy and antibiotics prior to BAL [5,9]. Quantitative bacterial and fungal cultures were performed according to generally accepted methods. Colony counts were determined, and identification obtained for each microorganism. In case of positive cultures, resistance to antimicrobial agents was tested. In 42 of 58 cases, BAL fluid was examined for $P$. jirovecii, and in 39 of 58 cases for CMV additionally. $P$. jirovecii antigen was diagnosed by a direct fluorescence antibody. For detection of CMV antigen and CMV DNA, direct immunofluorescence and polymerase chain reaction were performed, respectively. Aspergillus antigen and PCR have not been performed routinely. 
Table 1 Main clinical characteristics. Group A (neutrophile count $<1,000 / \mu 1$ ), group B (neutrophile count $>1,000 / \mu 1$ )

\begin{tabular}{|c|c|c|c|}
\hline & Group A & Group B & Overall \\
\hline Number of pneumonia & 30 & 28 & 58 \\
\hline Median age [years] of patients, (range) & $42(16-67)$ & $44(17-77)$ & $43(16-77)$ \\
\hline Number of female/ male & $8 / 22$ & $13 / 15$ & $21 / 37$ \\
\hline \multicolumn{4}{|l|}{ Number of patients with } \\
\hline Acute leukemia/ MDS & 12 & 3 & 15 \\
\hline Chronic myeloid leukemia & 1 & 6 & 7 \\
\hline Lymphoma, incl. CLL & 16 & 13 & 29 \\
\hline Solid tumor & 1 & 6 & 7 \\
\hline \multicolumn{4}{|l|}{ Number of previous chemotherapies } \\
\hline 1 & 21 & 10 & 31 \\
\hline $2-3$ & 6 & 15 & 21 \\
\hline$>3$ & 3 & 2 & 5 \\
\hline Autologous transplantation & 3 & 3 & 6 \\
\hline Allogeneic transplantation & 0 & 2 & 2 \\
\hline \multicolumn{4}{|l|}{ Median duration of neutropenia [days], (range) } \\
\hline Neutropenia $<1000 / \mu 1$ & $10(3-46)$ & & \\
\hline Neutropenia $<500 / \mu 1$ & $8.5(1-37)$ & & \\
\hline Number of patients with G-CSF & 22 & 4 & 26 \\
\hline \multicolumn{4}{|l|}{ Number of patients with } \\
\hline Antibacterial prophylaxis & 15 & 5 & 20 \\
\hline Antifungal prophylaxis & 4 & 1 & 5 \\
\hline Antiviral prophylaxis & 1 & 0 & 1 \\
\hline Median duration of prophylaxis [days], range & 11 & 22 & $12.5(3-190)$ \\
\hline Number of patients with antimicrobial first-line treatment started before BAL & 28 & 26 & 54 \\
\hline Median number of treatment regimes prior to BAL (range) & 2 & 1 & $1.5(0-5)$ \\
\hline Median duration of prior treatment [days], (range) & 6.5 & 6 & $6.5(1-28)$ \\
\hline
\end{tabular}

All data refer to the number of episodes of pneumonia

BAL fluid culture for bacteria is usually considered as clinically relevant if $\geq 10^{4} \mathrm{cfu} / \mathrm{ml}$ was detected. As BAL was performed under antimicrobial treatment in this study, and as it is known that antimicrobial treatment prior to BAL reduces the sensitivity of BAL cultures, we decided to consider bacterial cultures to be positive even if $<10^{4} \mathrm{cfu} /$ $\mathrm{ml}$ was detected.

The impact of bronchoscopy and BAL on treatment was evaluated from review of all progress notes and physician orders following the procedure. Treatment changes were recorded if they were made based on either a positive or negative result obtained from BAL. Therapeutic changes included addition and change of antimicrobial therapy when a pathogen was identified or discontinuation of empirical therapy when specific organisms were not found.

\section{Statistical analyses}

Data were analyzed by descriptive statistical methods using the statistical program SPSS. The Mann-Whitney $U$ test was used for unpaired, non-parametrical data, and chisquare test for comparison of qualitative variables. $P$ values $<0.05$ were considered significant.

\section{Results}

Fifty-eight episodes of pneumonia that underwent bronchoscopy and BAL due to clinically documented or suspected pneumonia were evaluable. Patients' median age was 43 years (range 16-77). Of 43 patients, one patient underwent four bronchoscopies, one patient three bronchoscopies, and ten patients had two bronchoscopies. In four patients, BAL was performed two times during the same episode of pneumonia, but with at least 6 days time interval. We considered these episodes as independent. In $88 \%$ of cases, patients had hematological diseases and in $12 \%$ of cases, patients had solid tumors (Table 1). Six patients underwent autologous hematopoietic stem cell transplantation and two had allogeneic stem cell transplan- 
tation in history. The most common underlying disease was non-Hodgkin lymphoma (Table 1). None of the patients had artificial ventilation before BAL. Of 30 patients with neutropenia, $26(87 \%)$ had at least neutropenia $<500 / \mu 1$. Very severe neutropenia $(<100 / \mu 1)$ occurred in $18(60 \%)$ of patients. Median duration of neutropenia was 10 days (range 3-46 days). Overall, $45 \%$ of patients received granulocyte colony stimulating factor (G-CSF), $73 \%$ in group A and $14 \%$ in group B.

In $93 \%$ of cases, BAL was performed under antimicrobial treatment, $93 \%$ in group A as well as in group B. In $40 \%$ of cases, patients had received antimicrobial prophylaxis before the beginning of infection, $57 \%$ in group $\mathrm{A}$ and $21 \%$ in group B. Thirty-five percent of patients received antibacterial prophylaxis (cotrimoxazole, colistin, levofloxacin, ofloxacin), 9\% antifungal prophylaxis (fluconazole, itraconazole), and $2 \%$ antiviral prophylaxis (aciclovir). The median duration of antimicrobial prophylaxis was 12.5 days (range 3-190 days). Fifty-one patients (88\%) received antimicrobial first-line treatment according to international guidelines [13, 16], 97\% of patients in group A and $79 \%$ in group B. These empirical regimen included ciprofloxacin plus amoxicillin-clavulanate, monotherapy with fourth-generation cephalosporin or carbapenem, antipseudomonal penicillin plus aminoglycoside and/or vancomycin, fourth-generation cephalosporin plus aminoglycoside and/or vancomycin, carbapenem plus aminoglycoside and/or vancomycin. Forty-one percent of patients received antifungal treatment as a part of the first-line treatment of pneumonia (amphotericin B, imidazole). In $50 \%$ of patients, antimicrobial treatment regimes were changed prior to BAL, up to five treatment regimes with a median of 1.5 treatment regimes were applied prior to BAL. Overall, the median duration of prior antimicrobial treatment (including first-line and following treatments due to any infection) was 6.5 days (range 1-28 days) with a median of 6.5 days in the group with neutropenia and 6 days in the group without neutropenia.

\section{Clinical results}

All patients had clinically documented pneumonia at time of BAL. According to the criteria of the American College of Chest Physicians and Society of Critical Care Medicine [20], in $71 \%$ of investigated periods, patients showed signs of sepsis, in $19 \%$, severe sepsis was diagnosed and $9 \%$ developed septic shock during the treatment period. Chest radiograph showed signs of infection in most cases, $93 \%$ in group A and $97 \%$ in group B. Overall, high-resolution CT was performed prior to BAL in 39 of $58(67 \%)$ cases to further characterize pulmonary alteration seen in chest radiograph or in cases of suspected pneumonia with negative results in chest radiograph. Radiological diagnosis of atypical pneumonia was made in 46 of 58 cases (79\%). P. jirovecii pneumonia and CMV pneumonia was suspected due radiological signs each in two cases.

Microbiological results

BAL revealed positive bacterial results in overall $67 \%$ of cases. There was no significant difference between neutropenic $(57 \%)$ and non-neutropenic $(79 \%)$ episodes $(p=$ 0.076). Gram-positive bacteria were the most common isolated microorganisms. Gram-positive microorganisms were detected in $95 \%$ of positive BAL results, gramnegative microorganisms in $23 \%$, mixed bacterial cultures occurred in 24 cases $(41 \%)$. The major pathogens detected were coagulase-negative staphylococci (80\%), Streptococcus species (51\%), Enterococcus species (18\%), Prevotella species (15\%), Pseudomonas aeruginosa (5\%), and Staphylococcus aureus (5\%; Table 2).

Only 17 of 39 (44\%) cases with positive BAL culture for bacteria showed at least $10^{4} \mathrm{cfu} / \mathrm{ml}$. If the cut-off was set at $\geq 10^{4} \mathrm{cfu} / \mathrm{ml}$, only in 17 of $58(29 \%)$ cases has a positive culture result been obtained, in group A $20 \%$ and in group B $39 \%$ of cases $(p=0.2)$. Among these, all positive cultures contained gram-positive bacteria; in $41 \%$ gram-negative bacteria were detected additionally. Accordingly, the main agents were coagulase-negative staphylococci (77\%), Streptococcus species (47\%), Enterococcus species (29\%), Prevotella species (24\%), and P. aeruginosa (12\%; Table 3).

Fungi were detected in $59 \%$ of cases. There was no significant difference between neutropenic and nonneutropenic patients $(p=0.9)$. All fungi detected were Candida species. Most frequent fungi were Candida albicans and Candida glabrata (each 50\%; Table 2). In four of 32 positive fungal cultures, more than one Candida species was detected.

In 39 cases, a CMV infection was suspected. Of these, $62 \%$ had a positive reaction of CMV antigen but in only $8 \%$ CMV PCR revealed a positive result, one case in group A and two cases in group B $(p=0.4)$. P. jirovecii antigen was detected in two of 42 cases tested, both cases appeared in the non-neutropenic group ( $p=0.1$; Table 4$)$.

Blood cultures revealed positive results in 10 of 58 cases $(17 \%), 27 \%$ in neutropenic patients, $7 \%$ in non-neutropenic patients. Seventy percent of bacteremia was due to grampositive bacteria and $30 \%$ due to gram-negative bacteria. No fungemia was detected. Only in one case of all patients with positive BAL results did blood cultures reveal a consistent positive result; in this case, in group $\mathrm{B} P$. aeruginosa was detected in both cultures.

Sputum cultures revealed positive results in 27 of 58 cases (47\%). In most cases (93\%), Candida species were detected; furthermore, in 17 cases, gram-positive bacteria and in five cases, gram-negative bacteria were detected. 
Table 2 Diagnostic yield of BAL in 58 episodes of pneumonia

In 24 of 39 positive cultures, more than one bacteria was detected. In four of 32 positive cultures, more than one fungus was detected

\begin{tabular}{|c|c|c|c|}
\hline & Group A & Group B & Overall \\
\hline Bacteria (total) & $17 / 30(57 \%)$ & $22 / 28(79 \%)$ & $39 / 58(67 \%)$ \\
\hline Gram-positive bacteria & $17 / 17(100 \%)$ & $20 / 22(91 \%)$ & $37 / 39(95 \%)$ \\
\hline Gram-negative bacteria & $1 / 17(6 \%)$ & $8 / 22(36 \%)$ & $9 / 39(23 \%)$ \\
\hline Coagulase-negative staphylococci & $16 / 17(94 \%)$ & $15 / 22(68 \%)$ & $31 / 39(80 \%)$ \\
\hline Streptococcus species & $10 / 17(59 \%)$ & $10 / 22(45 \%)$ & $20 / 39(51 \%)$ \\
\hline Enterococcus species & $1 / 17(6 \%)$ & $6 / 22(27 \%)$ & $7 / 39(18 \%)$ \\
\hline Pseudomonas aeruginosa & $1 / 17(6 \%)$ & $1 / 22(5 \%)$ & $2 / 39(5 \%)$ \\
\hline Stenotrophomonas maltophilia & 0 & $1 / 22(5 \%)$ & $1 / 39(3 \%)$ \\
\hline Klebsiella pneumoniae & 0 & $1 / 22(5 \%)$ & $1 / 39(3 \%)$ \\
\hline Staphylococcus aureus & 0 & $2 / 39(5 \%)$ & $2 / 39(5 \%)$ \\
\hline Haemophilus species & 0 & $1 / 39(3 \%)$ & $1 / 39(3 \%)$ \\
\hline Prevotella species & 0 & $6 / 22(27 \%)$ & $6 / 39(15 \%)$ \\
\hline Veillonella species & 0 & $3 / 22(14 \%)$ & $3 / 39(8 \%)$ \\
\hline Bifidobacterium adolescentis & 0 & $1 / 22(5 \%)$ & $1 / 39(3 \%)$ \\
\hline Fusobacterium nucleatum & 0 & $1 / 22(5 \%)$ & $1 / 39(3 \%)$ \\
\hline Fungus (total) & $17 / 29(59 \%)$ & $15 / 25(60 \%)$ & $32 / 54(59 \%)$ \\
\hline Candida albicans & $8 / 17(47 \%)$ & $8 / 15(53 \%)$ & $16 / 32(50 \%)$ \\
\hline Candida glabrata & $9 / 17(53 \%)$ & $7 / 15(47 \%)$ & $16 / 32(50 \%)$ \\
\hline Candida guilliermondii & $1 / 17(6 \%)$ & 0 & $1 / 32(3 \%)$ \\
\hline Candida krusei & 0 & $1 / 15(7 \%)$ & $1 / 32(3 \%)$ \\
\hline Candida parapsilosis & 0 & $1 / 15(7 \%)$ & $1 / 32(3 \%)$ \\
\hline Yeast (not differentiated) & 0 & $1 / 15(7 \%)$ & $1 / 32(3 \%)$ \\
\hline Aspergillus species & 0 & 0 & 0 \\
\hline
\end{tabular}

bacterial BAL results, for gram-positive as well as for gram-negative bacteria, were significantly less common in patients who underwent antibacterial prophylaxis than in patients who did not $(p=0.042)$. Neither the duration of empirical antimicrobial treatment nor the number of antimicrobial treatment regimes prior to BAL (started after onset of signs of infection) had a significant influence on a
Table 3 Diagnostic yield of BAL in 58 episodes of pneumonia

Only bacterial cultures with more than $10^{4} \mathrm{cfu} / \mathrm{ml}$ were regarded as relevant

\begin{tabular}{lrcc}
\hline & Group A & Group B & Overall \\
\hline Bacteria (total) & $6 / 30(20 \%)$ & $11 / 28(39 \%)$ & $17 / 58(29 \%)$ \\
Gram-positive bacteria & $6 / 6(100 \%)$ & $11 / 11(100 \%)$ & $17 / 17(100 \%)$ \\
Gram-negative bacteria & $1 / 6(17 \%)$ & $6 / 11(55 \%)$ & $7 / 17(41 \%)$ \\
Coagulase-negative staphylococci & $6 / 6(100 \%)$ & $7 / 11(64 \%)$ & $13 / 17(77 \%)$ \\
Streptococcus species & $2 / 6(33 \%)$ & $6 / 11(55 \%)$ & $8 / 17(47 \%)$ \\
Enterococcus species & $1 / 6(17 \%)$ & $4 / 11(36 \%)$ & $5 / 17(29 \%)$ \\
Pseudomonas aeruginosa & $1 / 6(17 \%)$ & $1 / 11(9 \%)$ & $2 / 17(12 \%)$ \\
Klebsiella pneumoniae & 0 & $1 / 11(9 \%)$ & $1 / 17(6 \%)$ \\
Staphylococcus aureus & 0 & $1 / 11(9 \%)$ & $1 / 17(6 \%)$ \\
Haemophilus species & 0 & $1 / 11(9 \%)$ & $1 / 17(6 \%)$ \\
Prevotella species & 0 & $4 / 11(36 \%)$ & $4 / 17(24 \%)$ \\
Veillonella species & 0 & $2 / 11(18 \%)$ & $2 / 17(12 \%)$ \\
Bifidobacterium adolescentis & 0 & $1 / 11(9 \%)$ & $1 / 17(6 \%)$ \\
Fusobacterium nucleatum & 0 & $1 / 11(9 \%)$ & $1 / 17(6 \%)$ \\
\hline
\end{tabular}


Table 4 Virological results and detection of Pneumocystis jirovecii: diagnostic yield of BAL in 58 episodes of pneumonia

\begin{tabular}{lrrc}
\hline & \multicolumn{1}{c}{ Group A } & \multicolumn{1}{l}{ Group B } & \multicolumn{1}{l}{ Overall } \\
\hline CMV antigen & $9 / 19(47 \%)$ & $15 / 20(75 \%)$ & $24 / 39(62 \%)$ \\
CMV DNA & $1 / 21(5 \%)$ & $2 / 17(12 \%)$ & $3 / 38(8 \%)$ \\
Pneumocystis jirovecii & $0 / 24$ & $2 / 18(11 \%)$ & $2 / 42(5 \%)$ \\
\hline
\end{tabular}

positive or negative bacterial result of the BAL $(p=0.2$ and $p=0.6$, respectively).

\section{Therapeutic impact of BAL}

Overall, BAL results directed a change of therapy in six of all cases (11\%), two in group A (7\%) and four in group B $(14 \%)$. In one case in group $\mathrm{A}$, the detection of CMV antigen in BAL led to the addition of ganciclovir to the antimicrobial treatment, in another case, a treatment with amphotericin B was begun due to the detection of $C$. albicans in the BAL. In one non-neutropenic patient, the detection of alpha-hemolytic streptococci in bacterial culture led to a change of therapy, a treatment with penicillin $G$ was started. In two non-neutropenic patients, the detection of $C$. albicans resulted in a change of therapy and in one patient, the detection of P. jirovecii in BAL fluid led to the addition of cotrimoxazole to the antimicrobial treatment. Focusing on bacteria, only in one of 58 cases did a positive bacterial culture cause a change of antimicrobial treatment. This patient belonged to group B.

Concerning P. jirovecii and CMV, in group A, only one patient was diagnosed with CMV pneumonia and in group $\mathrm{B}$, two patients were diagnosed with $P$. jirovecii pneumonia and another two patients with CMV pneumonia. So, in most patients with radiological signs of atypical pneumonia (89\%), P. jirovecii pneumonia and CMV pneumonia whose antimicrobial therapy is associated with intensive side effects especially for the hematopoietic system could be excluded via BAL.

Complications due to bronchoscopy and BAL occurred in four of 58 cases (7\%), 7\% in group A as well as in group B. Complications included hypotension, hypoxia, and pneumothorax. Overall, 11 patients (19\%) died during the study period, six in group A, five in group B. Of these patients, nine died due to infection. Overall mortality related to pneumonia was $16 \%$. There was no significant difference in mortality between neutropenic and nonneutropenic patients $(p=0.8)$. There was either no difference in mortality rate for cases with positive or negative BAL results $(p=0.3)$. In the group of patients that BAL results guided further antimicrobial therapy, one patient died due to an Aspergillus pneumonia that was detected post mortem.

\section{Discussion}

BAL is a helpful diagnostic tool to establish diagnosis and clarify the etiology of pneumonia. There exist some data that support a frequent use of BAL in immunocompromised patients with pulmonary infiltrates $[8,14,21]$. But, especially in severely ill patients with neutropenia, BAL is associated with a high rate of complications [2, 11, 30]. Therefore, the application of BAL in this setting should be considered carefully. This study highlights, in a critical way, the importance of BAL in patients with lung infiltrates and neutropenia.

The diagnostic yield of BAL published for neutropenic patients with pneumonia ranged between $13 \%$ and $53 \%$ [3, $5,9,11,21,27,30]$. Nearly all of these data were collected in retrospective studies.

In neutropenic patients with infection, prompt antimicrobial treatment is mandatory and it should not be delayed by any diagnostic procedure $[7,13,23]$. Additionally, most neutropenic patients with pneumonia receive antifungal drugs according to international guidelines $[16,18,19,26]$. It is known that previous antimicrobial treatment lowers the yield of BAL culture and is probably responsible for the low impact on treatment changes $[4,5,7,9,30]$. We could demonstrate as well, that positive bacterial BAL results were significantly less common in patients who underwent antibacterial prophylaxis than in patients who did not.

Concerning the patient collective, our results were comparable to the data of Cordonnier et al. [5] as we used similar definitions of the neutropenic and non-neutropenic subgroup. The diagnostic yield of BAL (53\%) was comparable to our study and did not differ significantly between the two subgroups. However, in their study, BAL results led to changes in therapy in more than one third of cases. In contrast, we observed a change of treatment in only $11 \%$ of cases. Saito et al. [27] emphasized the poor therapeutic usefulness of BAL in neutropenic patients with hematological diseases. Their BAL results were compared in seven patients with those at autopsy performed within 3 weeks of BAL. Overall, the diagnostic yield was low $(15 \%)$, only one etiologic diagnosis by BAL and autopsy corresponded. Azoulay et al. [2] even demonstrated that in critically ill hematological and oncological patients with acute respiratory failure, non-invasive strategies had a higher diagnostic yield than bronchoscopy and BAL. On the other hand, the studies of Dunagan et al. [8], Hummel et al. [14], and Peikert et al. [21] were in favor for the application of BAL in immunocompromised patients. They stated good diagnostic yield and frequent therapeutic implications in more than $50 \%$ of patients, although an impact on survival could not be demonstrated.

It is remarkable that in our study the results of neutropenic and non-neutropenic patients are comparable. 
The relatively slight difference between the two groups concerning BAL results and therapeutic implications of BAL may be due to the fact that even group B patients were considered as immunocompromised, according to the definition of Jain et al. [15]. For, even if they were not neutropenic at the time of BAL, most of them had undergone chemotherapy and experienced some episodes of neutropenia as well.

As BAL was performed in $93 \%$ of cases under antimicrobial treatment in both groups, we decided to consider bacterial cultures to be positive even if $<10^{4} \mathrm{cfu} /$ $\mathrm{ml}$ were detected [22]. In spite of this low cut-off in pretreated patients, the therapeutic consequences of bacterial BAL results remained rare, only one of the antimicrobial treatment changes in our study was due to results of bacterial cultures. This further supports the low impact of BAL on treatment. Similar in fungal pathogens, there was a high rate of positive cultures for Candida species. However, the probability of a Candida pneumonia is very low. Since Candida species belong to the physiological flora of the gastrointestinal tract, evidence of Candida in BAL has to be regarded as contamination until invasive disease is proven by lung biopsy [19, 26]. In our study Candida, pneumonia could not be proven in any case. Nevertheless, in three cases, a change of treatment was performed due to detection of Candida species that retrospectively had to be considered as clinically irrelevant. Knowing the disadvantages of a retrospective study, we abstained from evaluating the accuracy and probability of etiologic diagnosis, which had been made with the aid of BAL results. We did not evaluate if results were false positive or negative, but we evaluated the impact on antimicrobial treatment and we stated that the high amount of bacterial results, independent of the chosen cut-off, did not result in any treatment change. However, this cognition does not affect the importance of BAL in detecting or excluding $P$. jirovecii pneumonia or CMV pneumonia.

The bacterial spectrum isolated in our patients corresponded to the spectrum obtained in other studies in immunocompromised host with Streptococcus and Staphylococcus species isolated most frequently [7, 9, 11]. Most common organisms found in immunocompromised patients by Eriksson et al. [9] were alpha-hemolytic streptococci, coagulase-negative staphylococci, enterococci, and Neisseria species. P. aeruginosa was the most frequent gramnegative microorganism detected corresponding with other data in hematological malignancies [11]. But the significance of alpha-hemolytic streptococci, enterococci, and coagulase-negative staphylococci obtained from BAL culture remains unclear, since most of them occur as commensals and rarely cause pneumonia. Maschmeyer et al. [19] defined the isolation of these microorganisms from BAL fluid as etiologically insignificant findings for lung infiltrates. However, there are some authors suggesting that immunocompromised patients may develop pneumonia from these microorganisms $[7,17,28]$.

In our study, overall mortality related to pneumonia was $16 \%$. There was no difference in mortality rate for cases with positive or negative BAL results. As detection of microorganism in BAL did not lead to a relevant rate of antimicrobial treatment changes, BAL results had probably no influence on mortality. The rate of BAL-associated complications was very low in our study $(7 \%)$. This might be related to the retrospective character of the study and eventually missing documentation of minor complications.

\section{Conclusion}

BAL had a low impact on antimicrobial treatment. We summarize that the significance of BAL has decreased and its use should be considered with care in patients with pneumonia during neutropenia. However, this thesis based on a retrospective analysis should be tested in a prospective study. Nevertheless, we are convinced that BAL is still of high diagnostic value to distinguish between pulmonary tumor infiltration and pneumonic infiltration or to detect specific infections like $P$. jirovecii or CMV pneumonia.

\section{References}

1. Akova M, Paesmans M, Calandra T, Viscoli C, International Antimicrobial Therapy Group of the European Organization for Research and Treatment of Cancer (2005) A European organization for research and treatment of cancer - international antimicrobial therapy group study of secondary infections in febrile, neutropenic patients with cancer. Clin Infect Dis 40:239-245. doi:10.1086/426815

2. Azoulay E, Mokart D, Rabbat A, Pene F, Kouatchet A, Bruneel F, Vincent F, Hamidfar R, Moreau D, Mohammedi I, Epinette G, Beduneau G, Castelain V, de Lassence A, Gruson D, Lemiale V, Renard B, Chevret S, Schlemmer B (2008) Diagnostic bronchoscopy in haematology and oncology patients with acute respiratory failure: Prospective multicenter data. Crit Care Med 36:100-107. doi:10.1097/01.CCM.0000295590.33145.C4

3. Boersma WG, Erjavec Z, van der Werf TS, de Vries-Hosper HG, Gouw AS, Manson WL (2007) Bronchoscopic diagnosis of pulmonary infiltrates in granulocytopenic patients with hematologic malignancies: BAL versus PSB and PBAL. Respir Med 101:317-325. doi:10.1016/j.rmed.2006.04.021

4. Cantral DE, Tape TG, Reed EC, Spurzem JR, Rennard SI, Thompson AB (1993) Quantitative culture of bronchoalveolar lavage fluid for the diagnosis of bacterial pneumonia. Am J Med 95:601-607. doi:10.1016/0002-9343(93)90356-T

5. Cordonnier C, Escudier E, Verra F, Brochard L, Bernaudin JF, Fleury-Feith J (1994) Bronchoalveolar lavage during neutropenic episodes: diagnostic yield and cellular pattern. Eur Respir J 7:114-120. doi:10.1183/09031936.94.07010114

6. Crawford J, Dale DC, Lyman GH (2004) Chemotherapy-induced neutropenia: risks, consequences, and new directions for its management. Cancer 100:228-237. doi:10.1002/cncr.11882 
7. Dalhoff K, Braun J, Hollandt H, Lipp R, Wiessmann KJ, Marre R (1993) Diagnostic value of bronchoalveolar lavage in patients with opportunistic and non-opportunistic bacterial pneumonia. Infection 21:291-296. doi:10.1007/BF01712447

8. Dunagan DP, Baker AM, Hurd DD, Haponik EF (1997) Bronchoscopic evaluation of pulmonary infiltrates following bone marrow transplantation. Chest 111:135-141. doi:10.1378/ chest.111.1.135

9. Eriksson BM, Dahl H, Wang FZ, Elvin K, Hillerdahl G, Lundholm M, Linde A, Olding-Stenkvist E (1996) Diagnosis of pulmonary infections in immunocompromised patients by fiberoptic bronchoscopy with bronchoalveolar lavage and serology. Scand J Infect Dis 28:479-485. doi:10.3109/00365549609037944

10. Ewig S, Glasmacher A, Ulrich B, Wilhelm K, Schäfer H, Nachtsheim KH (1998) Pulmonary infiltrates in neutropenic patients with acute leukemia during chemotherapy. Chest 114:444-451. doi:10.1378/chest.114.2.444

11. Gruson D, Hilbert G, Valentino R, Vargas F, Chene G, Bebear C, Allery A, Pigneux A, Gbikpi-Benissan G, Cardinaud JP (2000) Utility of fiberoptic bronchoscopy in neutropenic patients admitted to the intensive care unit with pulmonary infiltrates. Crit Care Med 28:2224-2230. doi:10.1097/00003246-200007000-00007

12. Heussel CP, Kauczor HU, Heussel GE, Fischer B, Begrich M, Mildenberger P, Thelen M (1999) Pneumonia in febrile neutropenic patients and in bone marrow and blood stem cell transplant recipients: use of high resolution computed tomography. J Clin Oncol 17:796-805

13. Hughes WT, Armstrong D, Bodey GP, Bow EJ, Brown AE, Calandra T, Feld R, Pizzo PA, Rolston KV, Shenep JL, Young LS (2002) 2002 guidelines for the use of antimicrobial agents in neutropenic patients with cancer. Clin Infect Dis 34:730-751. doi:10.1086/339215

14. Hummel M, Rudert S, Hof H, Hehlmann R, Buchheidt D (2008) Diagnostic yield of bronchoscopy with bronchoalveolar lavage in febrile patients with hematologic malignancies and pulmonary infiltrates. Ann Hematol 87:291-297. doi:10.1007/s00277-0070391-6

15. Jain P, Sandur S, Meli Y, Arroliga AC, Stoller JK, Mehta AC (2004) Role of flexible bronchoscopy in immunocompromised patients with lung infiltrates. Chest 125:712-722. doi:10.1378/ chest.125.2.712

16. Link H, Bohme A, Cornely OA, Hoffken K, Kellner O, Kern WV, Mahlberg R, Maschmeyer G, Nowrousian MR, Ostermann H, Ruhnke M, Sezer O, Schiel X, Wilhelm M, Auner HW, Diseases Working Party (AGIHO) of the German Society of Hematology and Oncology (DGHO), Group Interventional Therapy of Unexplained Fever, Arbeitsgemeinschaft Supportivmassnahmen in der Onkologie (ASO) of the Deutsche Krebsgesellschaft(DKG-German Cancer Society) (2003) Antimicrobial therapy of unexplained fever in neutropenic patients - guidelines of the Infectious Diseases Working Party (AGIHO) of the German Society of Hematology and Oncology (DGHO), Study Group Interventional Therapy of Unexplained Fever, Arbeitsgemeinschaft Supportivmassnahmen in der Onkologie (ASO) of the Deutsche Krebsgesellschaft (DKGGerman Cancer Society). Ann Hematol 82(Suppl 2):105-117. doi:10.1007/s00277-003-0764-4

17. MacEachern P, Giannoccaro JP, Elsayed S, Read RR, Laupland KB (2005) A rare case of pleuropulmonary infection and septic shock associated with Enterococcus faecium endocarditis. J Infect 50:84-88. doi:10.1016/j.jinf.2003.11.005

18. Maschmeyer G, Link H, Hiddemann W, Meyer P, Helmerking M, Eisenmann E, Schmitt J, Adam D (1994) Pulmonary infiltrations in febrile patients with neutropenia. Risk factors and outcome under empirical antimicrobial therapy in a randomized multicenter study. Cancer 73:2296-2304. doi:10.1002/1097-0142(19940501) 73:9<2296::AID-CNCR2820730910>3.0.CO;2-7

19. Maschmeyer G, Beinert T, Buchheidt D, Einsele H, Heussel CP, Kiehl M, Lorenz J, Infectious Diseases Working Party (AGIHO) of the German Society of Hematology and Oncology (DGHO) (2003) Diagnosis and antimicrobial therapy of pulmonary infiltrates in febrile neutropenic patients-guidelines of the Infectious Diseases Working Party (AGIHO) of the German Society of Hematology and Oncology (DGHO). Ann Hematol 82(Suppl 2):118-126

20. Members of the American College of Chest Physicians/Society of Critical Care Medicine, consensus conference committee (1992) American College of Chest Physicians/Society of Critical Care Medicine consensus conference: Definitions for sepsis and organ failure and guidelines for the use of innovative therapies in sepsis. Crit Care Med 20:864-874

21. Peikert T, Rana S, Edell ES (2005) Safety, diagnostic yield, and therapeutic implications of flexible bronchoscopy in patients with febrile neutropenia and pulmonary infiltrates. Mayo Clin Proc 80:1414-1420. doi:10.4065/80.11.1414

22. Pereira Gomes JC, Pedreira WL, Araujo EM, Soriano FG, Negri EM, Antonangelo L, Tadeu Velasco I (2000) Impact of BAL in the management of pneumonia with treatment failure: positivity of BAL culture under antibiotic therapy. Chest 118:1739-1746. doi:10.1378/chest.118.6.1739

23. Pizzo PA (1993) Management of fever in patients with cancer and treatment-induced neutropenia. N Engl J Med 328:1323-1332. doi:10.1056/NEJM199305063281808

24. Ramila E, Sureda A, Martino R, Santamaria A, Franquet T, Puzo C, Montesinos J, Perea G, Sierra J (2000) Bronchoscopy guided by high-resolution computed tomography for the diagnosis of pulmonary infections in patients with hematologic malignancies and normal plain chest X-ray. Haematologica 85:961-966

25. Rano A, Agusti C, Jimenez P, Angrill J, Benito N, Danes C, Gonzalez J, Rovira M, Pumarola T, Moreno A, Torres A (2001) Pulmonary infiltrates in non-HIV immunocompromised patients: a diagnostic approach using non-invasive and bronchoscopic procedures. Thorax 56:379-387. doi:10.1136/thorax.56.5.379

26. Ruhnke M, Böhme A, Buchheidt D, Donhuijsen K, Einsele H, Enzensberger R, Glasmacher A, Gümbel H, Heussel CP, Karthaus M, Lambrecht E, Südhoff T, Szelenyi H (2003) Diagnosis of invasive fungal infections in hematology and oncology-guidelines of the Infectious Diseases Working Party (AGIHO) of the German Society of Hematology and Oncology (DGHO). Ann Hematol 82(Suppl 2):141-148. doi:10.1007/s00277-003-0768-0

27. Saito H, Anaissie EJ, Morice RC, Dekmezian R, Bodey GP (1988) Bronchoalveolar lavage in the diagnosis of pulmonary infiltrates in patients with acute leukemia. Chest 94:745-749. doi:10.1378/chest.94.4.745

28. Shenep JL (2000) Viridans-group streptococcal infections in immunocompromised hosts. Int J Antimicrob Agents 14:129 135. doi:10.1016/S0924-8579(99)00172-7

29. Sternberg RI, Baughman RP, Dohn MN, First MR (1993) Utility of bronchoalveolar lavage in assessing pneumonia in immunosuppressed renal transplant recipients. Am J Med 95:358-364. doi:10.1016/0002-9343(93)90303-7

30. Whittle AT, Davis M, Johnson PR, Leonard RC, Greening AP (1999) The safety and usefulness of routine bronchoscopy before stem cell transplantation and during neutropenia. Bone Marrow Transplant 24:63-67. doi:10.1038/sj.bmt.1701818

31. Wilhelm M, Kantarjian HM, O'Brien S, Pierce S, Keating MJ, Freireich EJ, Estey EH (1996) Pneumonia during remission induction chemotherapy in patients with AML or MDS. Leukemia 10:1870-1873 